\title{
Les écritures égéennes anciennes, déchiffrées et indéchiffrables
}

\section{Julien Zurbach}

\section{(2) OpenEdition}

1 Journals

Édition électronique

URL : http://journals.openedition.org/rbnu/1463

DOI : 10.4000/rbnu.1463

ISSN : 2679-6104

\section{Éditeur}

Bibliothèque nationale et universitaire de Strasbourg

\section{Édition imprimée}

Date de publication : 1 mai 2016

Pagination : 8-15

ISBN : 9782859230623

ISSN : 2109-2761

\section{Référence électronique}

Julien Zurbach, "Les écritures égéennes anciennes, déchiffrées et indéchiffrables », La Revue de la BNU [En ligne], 13 | 2016, mis en ligne le 01 mars 2020, consulté le 10 décembre 2020. URL : http:// journals.openedition.org/rbnu/1463; DOI : https://doi.org/10.4000/rbnu.1463

\section{(c) (i) (2)(2)}

La Revue de la BNU est mise à disposition selon les termes de la Licence Creative Commons Attribution - Pas d'Utilisation Commerciale - Partage dans les Mêmes Conditions 4.0 International. 
GRILLE SYLLABIOUE DU LINEAIRE B

WORK NOTE 15

(2* rédaction)

ANALYSE DES COMMBNAISONS DES CONSONNES ET OESS

VOYELLES DANS LE MATERIEL A FLEXION DE PYLOS :

ATHENES

28 SEPT. 61

\begin{tabular}{|c|c|c|c|c|c|}
\hline 党总 & 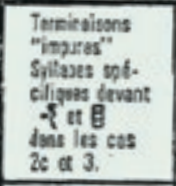 & 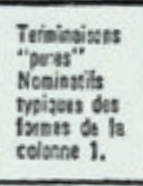 & $\begin{array}{l}\text { Avec } \\
\text { accusatils } \\
\text { possibles }\end{array}$ & \multicolumn{2}{|c|}{$\begin{array}{l}\text { Aussi, mais moins fréçem- } \\
\text { ment, les nominatils des } \\
\text { formes de la colonne } 1 .\end{array}$} \\
\hline 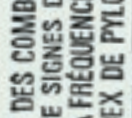 & $\begin{array}{l}\text { CES SIGKES } \\
\text { NAPPARRISSENT } \\
\text { PAS DEVANT-B- }\end{array}$ & \multicolumn{4}{|c|}{$\begin{array}{l}\text { CES SIGNES APPARAISSENT RAREMENT OU PAS } \\
\text { DU TOUT DEVANT - - }-\end{array}$} \\
\hline 象出崖 & $\begin{array}{l}\text { PLUS SOLVEAT } \\
\text { FEMININ QUE } \\
\text { MASCULIN? }\end{array}$ & \multicolumn{3}{|c|}{$\begin{array}{l}\text { PUS SOUVERT MASCULIM } \\
\text { OUE FEMININ? }\end{array}$} & $\begin{array}{l}\text { PLUS SOUVENT } \\
\text { FENININ OJE } \\
\text { MASCULIN? }\end{array}$ \\
\hline 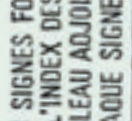 & \multicolumn{2}{|c|}{$\begin{array}{l}\text { FOZMENT KORMALEMENT } \\
\text { IE GENITIF SINGULIER EN } \\
\text { AJJUTANT }-\overline{7}\end{array}$} & \multicolumn{3}{|c|}{$\begin{array}{l}\text { IORMENT NOAMALENENT } \\
\text { IE GENITIF SINGULIER EN } \\
\text { A.UUTANT - ES }\end{array}$} \\
\hline 此 & Voyelle 1 & Voyeli: 2 & Voyelle 3 & Voyelle 4 & Voylle 5 \\
\hline
\end{tabular}

\begin{tabular}{|c|c|c|c|c|c|}
\hline wyelles & 530.3 & & & & $T^{4} 37.2$ \\
\hline $\begin{array}{l}\text { semi- } \\
\text { voyalle? }\end{array}$ & & & & 340 & Br 29.4 \\
\hline $\begin{array}{c}\text { consonas } \\
1\end{array}$ & A 14.8 & 32.5 & $2 \quad 21.2$ & 28.1 & $\Pi^{2} 18.8$ \\
\hline 2 & A 19.6 & 7 & & & $\mp \quad 13.7$ \\
\hline 3 & & 4 & & yt 35 & F 10.0 \\
\hline 4 & 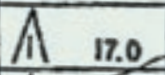 & 28.6 & & & iii. $\quad 0.4$ \\
\hline 5 & $A+17.7$ & $17 \quad 10.3$ & & $|\hat{v}|$ & $\gamma_{1} \quad 10.2$ \\
\hline 6 & $\bar{x}$ & A. 205 & & 2I5 14.8 & $\overline{\bar{p}}$ \\
\hline 8 & IS & 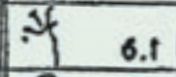 & & 12.5 & $43 \quad 15.2$ \\
\hline 9 & & 8 33.1 & & 32.3 & 2.4 \\
\hline 10 & $\frac{2}{2}$ & & 推 & 3.5 & f \\
\hline 11 & $n^{\leftarrow}-31.2$ & F 33.8 & ए 34.4 & $\mathrm{I}^{\mathrm{N}} \mathrm{a}$ & \# $\quad 0.7$ \\
\hline 12 & 17.0 & & & A 37.7 & $\theta \quad 24.0$ \\
\hline 13 & & $\frac{1 I}{I}$ & $8 \quad 14.2$ & & \\
\hline 14 & II & & & & \\
\hline 15 & $\sqrt{7} \quad 12.6$ & & & & \\
\hline
\end{tabular}

MICKAEL VEKTRIS

Figure I3. $^{-}$La grille de Ventris, 28-IX-I95I. 


\section{LES ÉGRITURES ÉGÉENNES ANGIENNES, DÉCHIFFRÉES ET INDÉCHIFFRABLES}

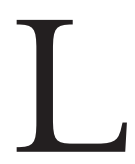

e déchiffrement du linéaire $B$ en 1952 a tout d'un roman. Architecte de formation, parlant plusieurs langues, Michael Ventris est parvenu à lire la forme la plus ancienne de grec qui fut écrite. Avant de décéder dans un accident de voiture en 1956, à 34 ans, il put publier, avec John Chadwick, un livre présentant les implications linguistiques et historiques de cette découverte majeure, faite contre les convictions dominantes, contre les siennes propres également, puisqu'il cherchait de l'étrusque dans ces textes. C'est un déchiffrement sans pierre de Rosette, qui a laissé le souvenir d'une démarche extrêmement systématique ; il n'appartient plus à l'ère romantique des hiéroglyphes égyptiens, mais bien à l'ère rationnelle $\mathrm{du} 2 \mathrm{O}^{\mathrm{e}}$ siècle, au point qu'une légende tenace mais jusqu'ici sans fondement attribue à Ventris des fonctions dans le déchiffrement des messages ennemis lors de son service militaire pendant et après la Seconde Guerre mondiale.

Les écritures anciennes, dites " égéennes ", sont bien antérieures à l'alphabet grec, en usage sur les rives de la mer Égée à partir du $8^{\mathrm{e}}$ siècle avant J.-C. Elles en sont aussi profondément différentes par bien des aspects. Écritures syllabiques et non alphabétiques, faisant un large usage d'idéogrammes, elles sont conservées sur des supports d'argile, et servent surtout à écrire des textes administratifs, inventaires, listes de tous types, rôles d'imposition, registres fonciers. Elles forment un groupe de trois écritures attestées en Crète et, pour la dernière, sur le continent grec. On les appelle écriture hiéroglyphique crétoise, linéaire A et linéaire B. Le groupe comprend aussi un rameau chypriote, qui comprend des écritures de l'âge du bronze mais aussi une écriture dite syllabaire chypriote classique - la seule parmi les égéennes à rester en usage après la fin de l'âge du bronze.

\section{La découverte des écritures égéennes}

C'est à l'archéologue autodidacte Heinrich Schliemann que l'on doit la découverte des grands sites de l'âge du bronze. Dans les années 1870, ses fouilles à Troie, Mycènes et Tirynthe révèlent l'existence de périodes très anciennes, qu'il conçoit comme l'âge des héros homériques. Ce moment essentiel, souvent évoqué en rapport avec les études homériques (la guerre de Troie a-t-elle eu lieu ?), doit se placer dans une autre perspective. La controverse fait rage, à la fin du $19^{e}$ siècle, sur l'existence même d'un âge du bronze, à placer entre la préhistoire ancienne, celle de la pierre taillée puis polie, et l'Antiquité gréco-romaine. En Égée, la question est résolue par les découvertes de Schliemann et toutes celles qui les suivent presque immédiatement. Elles sont un élément d'un mouvement qui donne corps à des périodes archéologiques de plus en plus anciennes dans ce berceau du monde grec classique. Les fouilles d'Olympie donnent corps à l'époque archaïque, à partir de 1875 ; le néolithique grec devient une réalité tangible au tournant du $20^{\mathrm{e}}$ siècle.

Dès ce moment, nombreux sont ceux qui se demandent si ceux qu'on appelle désormais les Mycéniens connaissaient une forme d'écriture. Arthur Evans, 


\section{Chronologie de l'âge du bronze égéen}

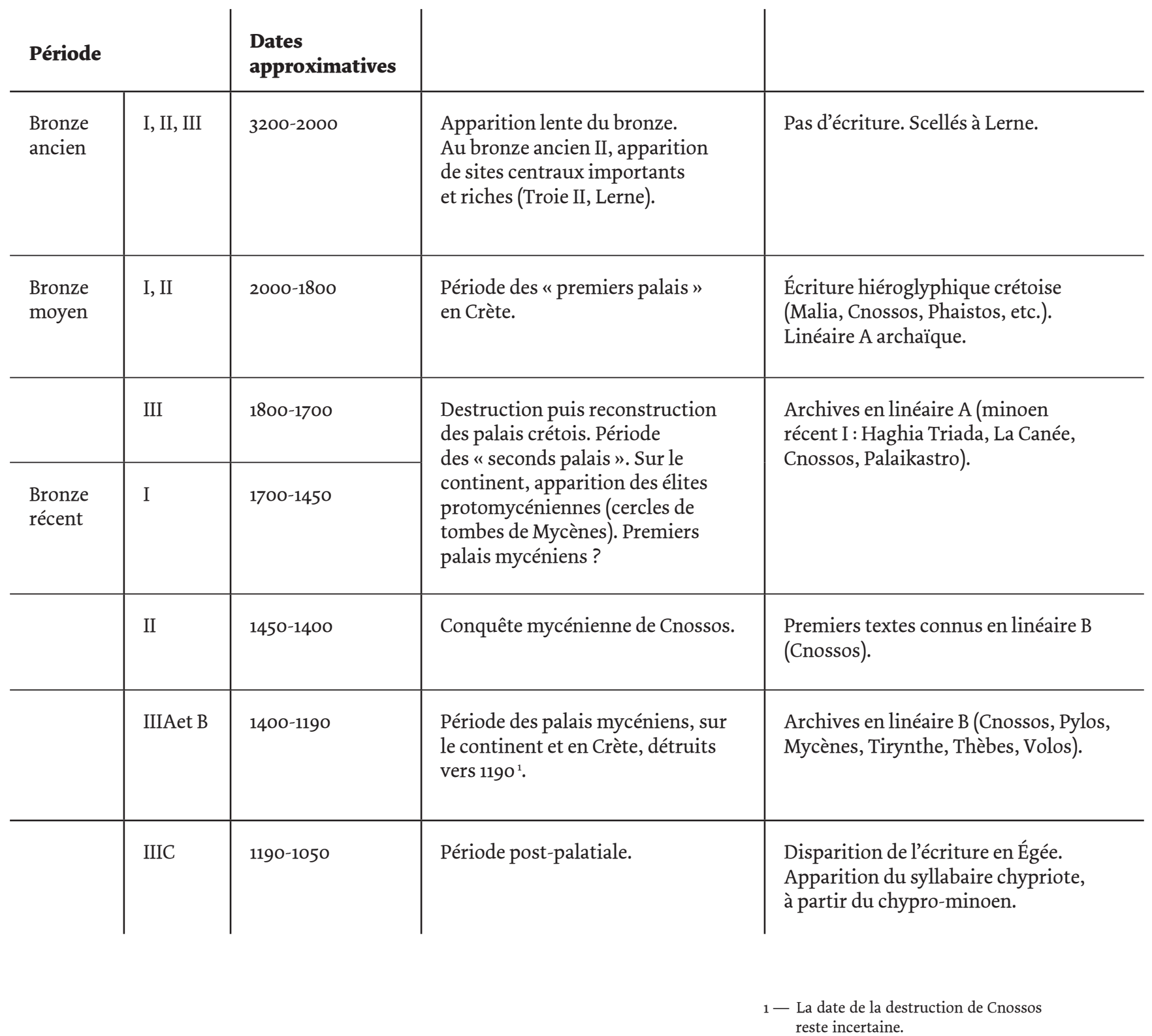


conservateur à l'Ashmolean Museum d'Oxford, en est persuadé : à partir de trouvailles éparses faites en Crète, il montre que des sceaux de l'âge du bronze ont porté des inscriptions dans une écriture inconnue. Puis, fouillant à Cnossos, en Crète, à partir de 1900, il met au jour dès la première semaine de fouille un ensemble de textes qui reste aujourd'hui le plus important du monde égéen. C'est à Evans qu'il revint de classer les trois systèmes d'écriture et de leur donner les noms que nous utilisons toujours aujourd'hui. Dans le premier volume de Scripta minoa, paru dès 1909, il identifiait sur critères externes l'écriture hiéroglyphique crétoise, l'écriture linéaire A et l'écriture linéaire $\mathrm{B}$. Ces termes se réfèrent à l'aspect des signes : plus pictographique pour la première qui, malgré son nom, n'a rien à voir avec les hiéroglyphes égyptiens ; plus schématique, linéaire, pour les deux autres. Evans ne tenta que quelques hypothèses sur des signes particuliers, sans s'attacher à un déchiffrement complet. Il était convaincu que ces trois écritures étaient purement crétoises et minoennes, et notaient une langue qui n'était pas du grec.

Ce sont les découvertes de Pylos, en Messénie, à partir de 1939, qui changèrent la donne en montrant que le linéaire B n'était probablement pas l'écriture de la chancellerie cnossienne qu'Evans avait cru y voir. On trouva ensuite, en 1950, des tablettes à Mycènes même. Mais ce n'est pas pour autant qu'on envisagea que ces textes pouvaient être en grec. Bien au contraire : lorsque Michael Ventris travaille au déchiffrement du linéaire B, il est d'abord convaincu que la langue notée est proche de l'étrusque, car c'est là, selon une idée répandue, la moins mal connue des langues qui, en Méditerranée orientale, auraient précédé le grec. Au printemps 1952, la logique interne de son système le pousse à admettre qu'il s'agit en fait d'une forme de grec très ancienne, et c'est cette hypothèse qu'il présente à la BBC en juin 1952, puis dans un article cosigné avec John Chadwick, paru dans le Journal of Hellenic Studies en 1953. C'est là le grand intérêt du déchiffrement de Ventris, comme on l'a souvent relevé. Alors que les tentatives antérieures - et nombre de celles qui aujourd'hui s'appliquent au linéaire A ou à d'autres documents - partaient d'un présupposé sur la nature de la langue notée et cherchaient ensuite, par diverses hypothèses, à trouver les valeurs phonétiques ou idéographiques des signes, Ventris adopte le chemin inverse. Nous verrons comment, et nous verrons aussi quelles furent les autres conditions indispensables au déchiffrement.

\section{Caractères de l'épigraphie égéenne}

Le matériel disponible est considérable, de par son importance historique, mais relativement restreint si on le compare à d'autres ensembles contemporains. Les inscriptions montrent une claire succession des écritures. Le hiéroglyphique crétois est attesté pour l'essentiel au $18^{\mathrm{e}}$ siècle avant notre ère, et presque exclusivement en Crète. Il est utilisé par l'administration de ce que nous appelons les premiers palais crétois, datant du minoen moyen. Le linéaire A est attesté, sous une forme archaïque, dès cette époque, ce qui pose le problème de l'existence contemporaine de deux systèmes d'écriture. Il est utilisé jusqu'à la fin des seconds palais crétois, vers 1450 . L'essentiel des documents date de cette période (minoen moyen III - minoen récent I) ; un seul, pour le moment, semble être nettement plus récent, la statuette de Poros, qui pourrait dater du $14^{\mathrm{e}}$ siècle. Le linéaire $B$ est une adaptation du linéaire $\mathrm{A}$ à la langue grecque, effectuée à partir d'une version du linéaire A que nous n'avons plus, peut-être celle des documents sur parchemin ou papyrus, et dans un lieu que nous ignorons. Cette adaptation répondait aux besoins nouveaux des principautés mycéniennes, qui se développent sur le continent alors que les seconds palais fonctionnent en Crète : les cercles de tombes de Mycènes sont la trace archéologique la plus connue de cette émergence de pouvoirs nouveaux sur le continent. Les plus anciens textes en linéaire B datent probablement de la fin du $15^{\mathrm{e}}$ siècle et sont dus à l'administration mycénienne installée à Cnossos après la conquête de la Crète centrale. Les plus récents, et les plus nombreux, datent des destructions qui mirent fin aux palais mycéniens, vers 119o. La date des textes de Cnossos reste un sujet débattu mais elle se situe en tout cas entre le milieu du $14^{\mathrm{e}}$ et le milieu du $13^{\mathrm{e}}$ siècle avant notre ère.

Pour donner un ordre d'idées, nous avons quelques centaines de documents en hiéroglyphique, tous assez courts; environ un millier en linéaire A; et environ 6000 en linéaire $\mathrm{B}$. Pour le linéaire $\mathrm{B}$, les tablettes découvertes à Cnossos à partir de 1900 sont environ 4 500. Il y a près de 900 textes à Pylos, quelques dizaines à Mycènes et Tirynthe, et environ 400 à Thèbes. C'est peu de choses si l'on songe au demi-million de tablettes cunéiformes conservées.

L'immense majorité des textes (et les plus longs) sont des documents administratifs, écrits sur des tablettes d'argile qui n'ont été conservées que parce qu'elles ont été cuites par accident, en général lors de la destruction des palais. L'écriture hiéroglyphique est aussi employée sur 
des sceaux, et le linéaire A comme le linéaire B se trouvent assez souvent sur des vases, notamment des vases de stockage ou de transport. Les sceaux hiéroglyphiques, et quelques inscriptions religieuses en linéaire $\mathrm{A}$, constituent les seules exceptions à l'usage strictement administratif de l'écriture. Il y eut probablement des documents sur support souple, parchemin ou papyrus. Des scellés portant la trace de documents de parchemin roulés ont été trouvés, notamment dans des contextes appartenant à la période des seconds palais, donc du linéaire A. Les textes mycéniens sur argile n'offrent quant à eux que la comptabilité courante, sur la dernière année ; des mentions de dettes de l'année précédente laissent supposer qu'il existait des registres plus complets, sur un autre support, et donc perdus pour nous. Quoi qu'il en soit, rien ne permet de supposer que ces parchemins aient porté autre chose que des textes administratifs ; rêver à une Iliade en linéaire $\mathrm{B}$ est évidemment permis, mais ne repose sur rien, pas plus que de supposer des lettres diplomatiques ou des annales royales, telles qu'on en trouve chez les Hittites et au Proche-Orient.

\section{Le déchiffrement du linéaire $B$}

De la nature des textes découle un premier obstacle au déchiffrement : il n'y a pas de document bilingue. Les relations extérieures, nombreuses, des Égéens n’ont pas donné lieu à l'établissement d'une pierre de Rosette. C'est donc par des considérations internes qu'il fallait parvenir à lire le linéaire B. La plupart des tentatives antérieures à Ventris partaient d'une hypothèse sur la langue écrite en linéaire $B$, en général ce qu'on pouvait penser être proche des langues parlées en Égée avant le grec (anatolien, basque, étrusque).

Ventris part au contraire d'un raisonnement sur la nature de l'écriture et non de la langue. Le nombre de signes phonétiques, environ 80 dans chacune des écritures, indique qu'il s'agit non d'un alphabet, mais d'un syllabaire, et d'un syllabaire probablement de type léger. Cela signifie que, comme le syllabaire chypriote, le linéaire B note des syllabes ouvertes (consonne et voyelle : CV) et n'a pas de signes notant des combinaisons plus complexes (VC, CVC, etc.), ce qui exige plusieurs centaines de signes, comme en cunéiforme.

Dans l'analyse du système d'écriture, deux contributions furent déterminantes. Alice Kober parvint à établir des alternances entre signes. En partant de questions précises (la langue a-t-elle une flexion ?), elle mit en série des mots qui se terminaient parfois par un signe, parfois par un autre. On pouvait raisonnablement penser que les signes en alternance avaient la consonne en commun, mais différaient par la voyelle de la désinence. D’autres alternances pouvaient être repérées en début de mot notamment. C'est par cette voie que peu à peu Ventris put construire une grille fondée sur les relations entre les signes (voyelles communes, consonnes communes), avant même de faire quelque hypothèse que ce soit sur la valeur de ces derniers. La grille était pour l'essentiel constituée en 1951 (voir ill. p. 8). Il restait à trouver comment insérer des valeurs consonantiques et vocaliques pour rendre possible la lecture des signes : l'exercice serait bien facilité par l'existence de la grille, mais restait difficile. Dans la work note 19, en mars 1952, Ventris cherche des parallèles étrusques, sans grand succès. C'est la work note $20, \mathrm{du}^{\mathrm{er}}$ juin 1952, qui l'amène à prendre au sérieux l'hypothèse grecque, alors qu'elle va contre l'opinion courante. Il prend comme point de départ l'idée que les mots les plus courants dans les tablettes de Cnossos sont probablement des toponymes. Il y avait là non seulement un argument de fréquence relative, mais aussi des considérations sur l'économie interne des textes, facilitées par le parallèle avec les écrits administratifs d'Ougarit - ces termes se retrouvaient souvent en début de texte. On disposait de textes de Pylos et Cnossos : cela permettait d'identifier comme probables toponymes les mots les plus courants qui ne se trouvaient que sur un de ces deux sites. En tentant de reconnaître Amnisos, Cnossos et Tylissos, noms connus par Homère, et en s'appuyant sur les analogies formelles les plus assurées avec le syllabaire chypriote, Ventris arrive à proposer des valeurs pour quelques signes. Ceci n'est pas nouveau : encore faut-il étayer l'hypothèse, et un toponyme peut se retrouver dans des langues bien différentes. Mais c'est là que l'effet conjugué de la grille et d'une analyse serrée des textes joue pleinement : très vite apparaissent des mots qu'on peut lire par le grec, tels ko-wo et ko-wa (pour des formes anciennes de kouros et korè) ou, surtout, to-so et to-sa indiquant des totaux.

C'est cela que Ventris présente à la $\mathrm{BBC}$ le $1^{\mathrm{er}}$ juillet 1952. La collaboration avec l'helléniste John Chadwick permet d'avancer vite vers un vocabulaire du grec mycénien qui, un mois plus tard, compte environ 500 mots. L'article publié dans le Journal of Hellenic Studies au printemps 1953 donne les principes du déchiffrement, les règles d'orthographe, l'analyse de quelques textes. C'est au printemps 1953 aussi que vint la preuve définitive. Une tablette, découverte à Pylos en 1952, put être lue 
par l'archéologue Blegen. Il s'agissait d'un inventaire de vases (appelé aujourd'hui PY Ta 641). Non seulement la plus grande partie des termes se comprenaient en grec, ce qui confirmait que le déchiffrement fonctionnait avec des textes inconnus lors de son élaboration ; mais en plus l'inventaire comporte des idéogrammes très précis, indiquant notamment par le dessin le nombre d'anses ou de pieds de chaque vase, et le texte décrit lui aussi ces caractères : on trouve $a-n o-w e$, qui signifie en grec " sans anses ", à côté du dessin d'un vase sans anses, ou ti-ri-po-de, "trépied ", à côté d'un vase à trois pieds. À partir de ce moment, la grande majorité des spécialistes acceptèrent le déchiffrement.

Le talent de Ventris est évident et indéniable. Il est certainement lié à son don pour les langues, plus sans doute qu'à une douteuse expérience dans le déchiffrement pendant la guerre. Mais il faut aussi insister sur d'autres aspects, proprement scientifiques, de son travail. Du début à la fin, Ventris travailla de manière collective. On a vu le rôle des recherches menées par Alice Kober. Ventris adressa en 1950 à plusieurs spécialistes reconnus, dans différents pays, un document faisant le point sur les connaissances relatives au linéaire B. Il leur adressa ensuite ses work notes, tout en entretenant avec chacun d'eux une correspondance suivie.

Ce caractère collectif s'illustre surtout par l'importance des travaux d'Emmett Bennett, l'épigraphiste de la mission américaine de Pylos. Bennett avait soutenu sa thèse en 1947 sur les textes en linéaire B de ce site, puis avait publié ces tablettes en 1951 sous forme de dessins. Il avait ainsi effectué un travail fondamental sur deux points. Les textes, tout d'abord, étaient disponibles. C'était le plus important ensemble mis à disposition de la recherche, alors que l'on ne disposait que d'un échantillon de textes de Cnossos, Scripta minoa II paraissant en 1952, un peu trop tard pour le déchiffrement. L'écriture, ensuite, avait été correctement analysée par Bennett, qui avait distingué clairement les idéogrammes des syllabogrammes, et bien identifié les variantes d'un signe. Il est en effet toujours difficile, quand on ne lit pas une écriture, de distinguer précisément entre deux signes proches et deux variantes d'un même signe.

La qualité des textes disponibles, ainsi que la précision de l'analyse du système graphique en amont, furent des éléments déterminants. Aucun déchiffrement n'est possible sans ces étapes fondamentales. Et le déchiffrement lui-même permit un retour aux textes : Ventris et Chadwick virent eux-mêmes ceux de Pylos, Cnossos et Mycènes entre 1953 et 1955, et c'est ce travail sur les textes qui leur permit de publier très vite un volume fondamental, toujours utilisé aujourd'hui, intitulé Documents in Mycenaean Greek (1955). L'esprit de collaboration internationale, qui rendait possibles ces publications rapides, s’incarna en avril 1956 dans le premier colloque d'études mycéniennes, organisé à Gif-sur-Yvette par Pierre Chantraine et Michel Lejeune.

\section{Comment aborder les textes en linéaire A ?}

Le linéaire $A$ est un système très proche du linéaire $B$, qui en est, comme on l'a vu, une adaptation. Il est donc tentant, et cela a été fait à plusieurs reprises, de simplement attribuer aux signes du linéaire A les valeurs phonétiques qui sont celles de leurs correspondants - leurs "descendants" - en linéaire B. Mais deux ordres de faits incitent à la plus grande prudence sur ce point. Il s'agit d'abord d'une expérience historique. Dans les tentatives de déchiffrement du linéaire $B$ antérieures aux travaux de Ventris, on accordait régulièrement un rôle important au syllabaire chypriote classique. C'était légitime : les valeurs des signes avaient été sûrement établies, dès le $18^{e}$ siècle avant J.-C., grâce à des inscriptions digraphes donnant un même texte grec en alphabet et en syllabaire. L'écriture étant visiblement apparentée au linéaire B, on pouvait penser qu'elle allait donner une clé de lecture essentielle. Mais l'écriture chypriote classique n'est qu'un lointain parent du linéaire $\mathrm{B}$, et les correspondances formelles entre signes de chaque écriture sont parfois difficiles à établir. Et surtout, les règles orthographiques ne sont pas les mêmes : en chypriote, un -s final s'écrit avec le signe se, la voyelle de ce dernier restant ignorée ; en linéaire $\mathrm{B}$, une consonne finale est le plus souvent ignorée et reste non transcrite (ícós, "sacré", s'écrit i-je-ro-se en chypriote, i-je-ro en linéaire B).

Ce procédé fondé sur les correspondances formelles est donc très dangereux. Pour une part, il revient à tenter de lire du grec en partant d'une forme ou d'une autre de cyrillique. L'application de la méthode de la grille au corpus du linéaire $\mathrm{A}$ a d'autre part mené à la conclusion que les signes ${ }^{*} 78$ et ${ }^{*} 80$ de cette écriture devaient partager soit la voyelle soit la consonne. Or ils se lisent en linéaire B ma et qe (c'est-à-dire $k^{w} e$ ). Ceci, évidemment, rend difficile d'appliquer les valeurs du linéaire B au linéaire A. Ces mêmes travaux ont par ailleurs montré que la grille du linéaire A reste pour le moment assez vide. C'est tout simplement que le nombre de " mots" connus, de suites de signes qu'on peut comparer, est 


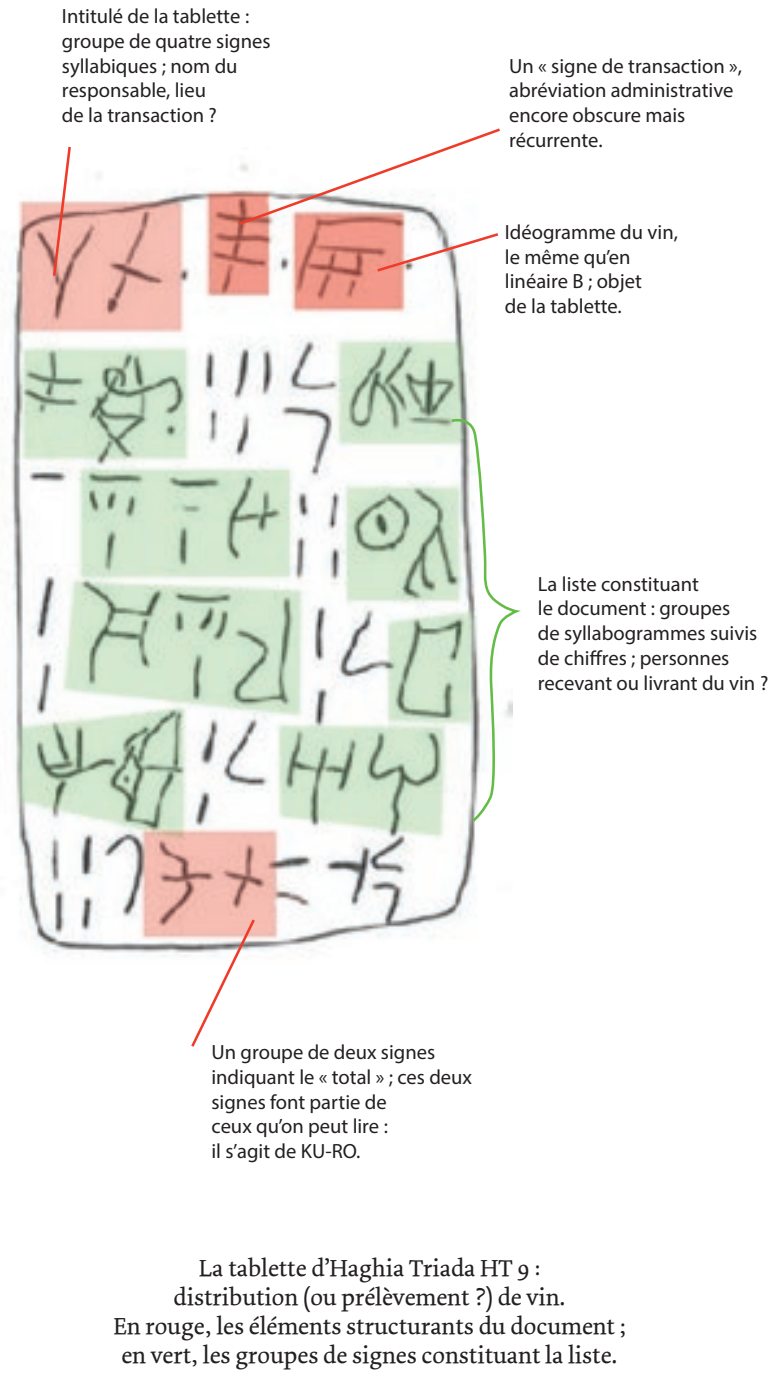

beaucoup plus réduit que celui du linéaire $B$ à l'époque où travaillait Ventris. Les éditeurs du Recueil des inscriptions en linéaire A, ouvrage de référence paru entre 1974 et 1985 , ont chacun tenté d'évaluer la situation au terme de leurs travaux. L. Godart conclut que 12 signes du linéaire A sur 85 syllabogrammes peuvent être lus avec un certain degré de certitude ; J.-P. Olivier arrive au même nombre. C'est donc une toute petite minorité. Le système syllabique du linéaire A ne pourra être mieux compris que lorsque le nombre d'inscriptions connues, et correctement publiées, aura augmenté de manière très nette.

À cela s'ajoute une autre difficulté. Le déchiffrement du linéaire B a été facilité par la présence d'une langue connue, même si le dialecte grec en question ne l'était pas auparavant. Tout indique que ce ne sera pas le cas en linéaire $\mathrm{A}$, et donc que, même lorsqu'on pourra attribuer des valeurs phonétiques aux signes de cette écriture, on ne pourra pas encore comprendre les mots et les textes. On se retrouvera, en un certain sens, dans la situation de l'étrusque, écrit dans un système syllabique qui permet de le lire, sans pour autant que la langue nous soit parfaitement compréhensible. Un exemple le montre bien. Parmi les signes dont la valeur phonétique est à peu près assurée se trouvent ceux qui composent le mot ku-ro, dont la place dans quelques tablettes montre qu'il signifie " total ". Ce terme n'a pas de parallèle évident ; on a tenté de l'expliquer par la racine sémitique de l'arabe kûl, hébreu kol, mais ce n'est pas assuré. Le même filon linguistique ne permet pas d'expliquer le terme po-to-kuro, " grand total ». En tout cas, ce n'est pas du grec, et aucun rapport avec une langue plus ou moins connue ne semble s'imposer. Il existe bien une langue, attestée entre le $7^{\mathrm{e}}$ et le $2^{\mathrm{e}}$ siècle avant J.-C., que nous appelons l'étéocrétois et qui est le meilleur candidat au statut de descendant d'une langue minoenne. Mais les quelques inscriptions de Dréros et Praisos qui sont attribuées à cette langue, écrites dans la variante locale de l'alphabet grec, sont courtes et obscures. Il n'est pas assuré qu'un lien existe entre la ou les langues notées en linéaire A d'une part, et l'étéocrétois de l'autre. Homère parle de la Crète comme d'une île multilingue (ainsi Odyssée, XIX, 175-177). Mais même si ce lien existait, il ne serait d'aucune aide dans le déchiffrement du linéaire A.

L'étude des syllabogrammes et du système phonétique du linéaire A ne permet donc pas d'entrevoir un prochain déchiffrement, ceci avant tout à cause du manque de documents. Mais cela ne signifie pas que les documents actuellement connus nous restent entièrement obscurs, comme des séries de traits absurdes. Les autres composantes du système d'écriture permettent d'approcher le contenu de ces textes. Il s'agit d'abord du système numéral. Le système des unités est identique en linéaire $A$ et en linéaire $B$ : un trait vertical pour 1 , un trait horizontal pour 10 , un cercle pour 10o. Mais là où le linéaire $B$ utilise une série d'unités de volume des liquides et des solides, le linéaire A utilise des fractions, dont le système a été tôt identifié, avant même le déchiffrement du linéaire $B$, même si bien des aspects en restent encore obscurs.

Les idéogrammes en linéaire A sont, comme en linéaire $B$, souvent de véritables idéogrammes, des 


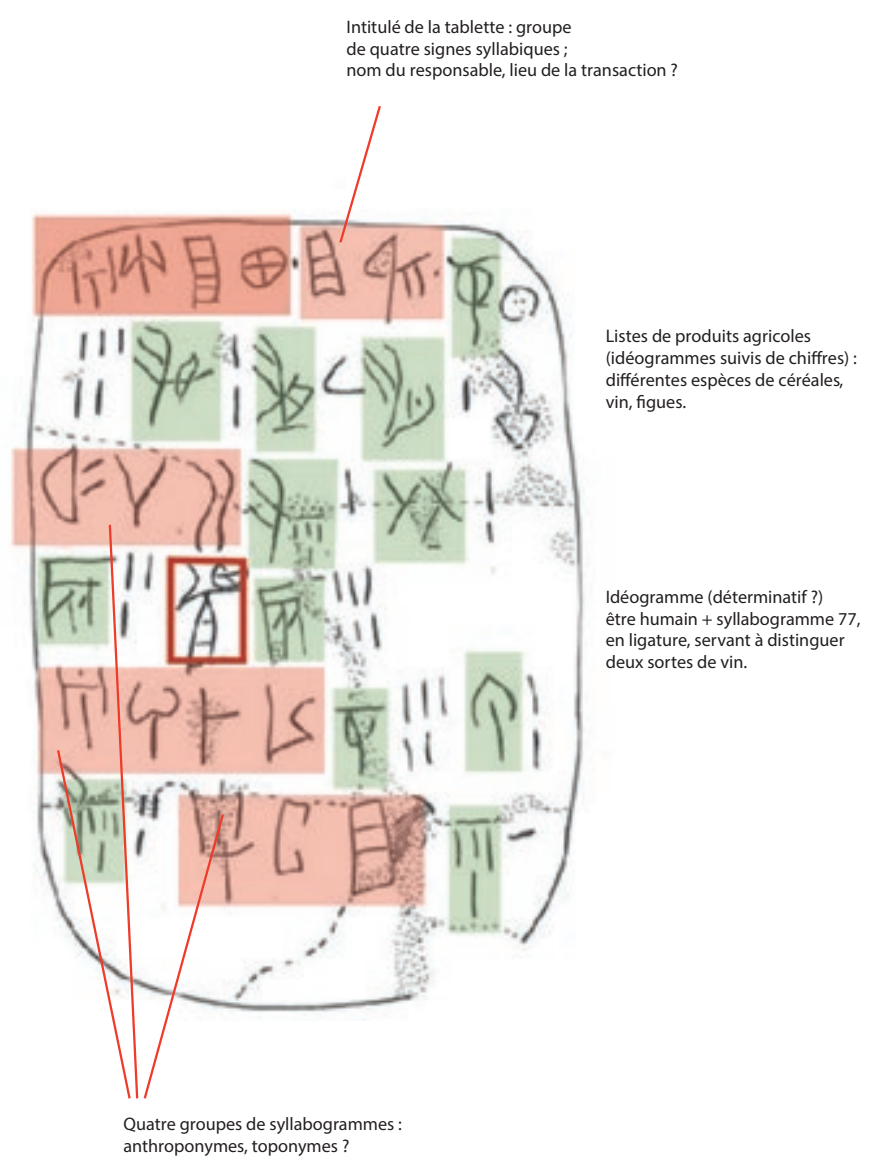

La tablette HT 28 : un document fiscal ?

dessins qui représentent clairement un objet. Tous ne sont pas de lecture si évidente, mais les principaux ont été directement empruntés par les inventeurs du linéaire B. Or si, dans le cas des syllabogrammes, il s'agit d'une question de système, et qu'on peut envisager que toute une série de syllabogrammes ait changé de valeur en fonction des besoins de la nouvelle langue à noter, dans le cas des idéogrammes, il y a peu de raison de changer la valeur du signe plutôt que d'en créer un nouveau. Un indice de ce conservatisme vient des idéogrammes qui sont en fait des abréviations. L'idéogramme de la figue en linéaire $B$ est $C$, qui est aussi le syllabogramme ni. En linéaire B, ce n'est pas cohérent : la figue en grec se dit sukon. Mais il existe un vieux mot dialectal crétois, conservé par le lexicographe Hésychius : nikuleon, qui signifie aussi " figue ". Il est possible qu'il s'agisse là d'une forme du mot courant en linéaire A, qui explique le choix du signe $n i$, dont on pourrait alors en retour considérer qu'il a la même valeur en linéaire A et B. Ce genre de cas est peu courant; mais on peut considérer que la signification des idéogrammes a une forte tendance à se maintenir d'une écriture à l'autre. C'est là un point de départ solide pour tenter d'interpréter les textes en linéaire A, pour la plupart des textes économiques formés de groupes de syllabogrammes, d'idéogrammes et de chiffres. Deux exemples sont donnés ci-contre.

Toutes ces observations partielles n'ont de sens que si elles permettent une approche fructueuse de textes qui restent non déchiffrés au sens où nous ne pouvons pas attribuer de valeur phonétique aux syllabogrammes. C'est là ce qui reste la grande limite de notre connaissance du linéaire $\mathrm{A}$ : face aux quelques textes de dédicace, par exemple, nous pouvons repérer des formules mais sommes incapables de dire s'il s'agit du nom de la divinité, d'une forme verbale ou d'une formule sacrée.

\section{Julien Zurbach}

\section{ORIENTATIONS BIBLIOGRAPHIQUES :}

— Chadwick, John, Le déchiffrement du linéaire B, Paris, 1972 (éd. orig. 1958)

- Duhoux, Yves, L'étéocrétois. Les textes, la langue, Amsterdam, 1982

— Godart, Louis, " La scrittura Lineare A ", in La parola del passato, 31, 1976, p. 30-47

— Godart, Louis, Olivier, Jean-Pierre, Recueil des inscriptions en linéaire A, I-V, Athènes-Paris, 1974-1985

- Olivier, Jean-Pierre, "'Lire' le linéaire A ? ", in J. Bingen, G. Cambier et G. Nachtergael (éd.), Le monde grec. Pensée, littérature, histoire, documents. Hommages à Claire Préaux, Bruxelles, 1975, p. 441-449

— Ventris, Michael et Chadwick, John, Documents in Mycenaean Greek, Cambridge, 1956, $2^{\mathrm{e}}$ éd. 1973 